\title{
Basic Properties and Concept of Selected Subsequence of Zero Based Finite Sequences
}

\author{
Yatsuka Nakamura \\ Shinshu University \\ Nagano, Japan
}

\author{
Hisashi Ito \\ Shinshu University \\ Nagano, Japan
}

\begin{abstract}
Summary. Here, we develop the theory of zero based finite sequences, which are sometimes, more useful in applications than normal one based finite sequences. The fundamental function $\mathrm{Sgm}$ is introduced as well as in case of normal finite sequences and other notions are also introduced. However, many theorems are a modification of old theorems of normal finite sequences, they are basically important and are necessary for applications. A new concept of selected subsequence is introduced. This concept came from the individual Ergodic theorem (see [7]) and it is the preparation for its proof.
\end{abstract}

MML identifier: $\underline{\text { AFINSQ_2, }}$, version: $\underline{7.9 .03 \quad 4.104 .1021}$

The articles [12], [1], [14], [5], [8], [2], [6], [4], [3], [13], [10], [9], and [11] provide the notation and terminology for this paper.

\section{Preliminaries}

In this paper $D$ is a set.

One can prove the following proposition

(1) For every set $x$ and for every natural number $i$ such that $x \in i$ holds $x$ is an element of $\mathbb{N}$.

Let us observe that every natural number is natural-membered. 


\section{Additional Properties of Zero Based Finite Sequence}

One can prove the following propositions:

(2) For every finite natural-membered set $X_{0}$ there exists a natural number $m$ such that $X_{0} \subseteq m$.

(3) Let $p$ be a finite 0 -sequence and $b$ be a set. If $b \in \operatorname{rng} p$, then there exists an element $i$ of $\mathbb{N}$ such that $i \in \operatorname{dom} p$ and $p(i)=b$.

(4) Let $D$ be a set and $p$ be a finite 0 -sequence. Suppose that for every natural number $i$ such that $i \in \operatorname{dom} p$ holds $p(i) \in D$. Then $p$ is a finite 0 -sequence of $D$.

The scheme $X S e q L a m b d a D$ deals with a natural number $\mathcal{A}$, a non empty set $\mathcal{B}$, and a unary functor $\mathcal{F}$ yielding an element of $\mathcal{B}$, and states that:

There exists a finite 0 -sequence $z$ of $\mathcal{B}$ such that len $z=\mathcal{A}$ and for every natural number $j$ such that $j \in \mathcal{A}$ holds $z(j)=\mathcal{F}(j)$

for all values of the parameters.

One can prove the following proposition

(5) Let $p, q$ be finite 0 -sequences. Suppose len $p=\operatorname{len} q$ and for every natural number $j$ such that $j \in \operatorname{dom} p$ holds $p(j)=q(j)$. Then $p=q$.

Let $f$ be a finite 0 -sequence of $\mathbb{R}$ and let $a$ be an element of $\mathbb{R}$. Then $f+a$ is a finite 0 -sequence of $\mathbb{R}$.

We now state two propositions:

(6) Let $f$ be a finite 0 -sequence of $\mathbb{R}$ and $a$ be an element of $\mathbb{R}$. Then len $(f+$ $a)=\operatorname{len} f$ and for every natural number $i$ such that $i<\operatorname{len} f$ holds $(f+a)(i)=f(i)+a$.

(7) For all finite 0 -sequences $f_{1}, f_{2}$ and for every natural number $i$ such that $i<\operatorname{len} f_{1}$ holds $\left(f_{1} \frown f_{2}\right)(i)=f_{1}(i)$.

Let $f$ be a finite 0 -sequence. The functor $\operatorname{Rev}(f)$ yielding a finite 0 -sequence is defined as follows:

(Def. 1) len $\operatorname{Rev}(f)=\operatorname{len} f$ and for every element $i$ of $\mathbb{N}$ such that $i \in \operatorname{dom} \operatorname{Rev}(f)$ holds $(\operatorname{Rev}(f))(i)=f(\operatorname{len} f-(i+1))$.

We now state the proposition

(8) For every finite 0 -sequence $f$ holds $\operatorname{dom} f=\operatorname{dom} \operatorname{Rev}(f)$ and $\operatorname{rng} f=$ rng $\operatorname{Rev}(f)$.

Let $D$ be a set and let $f$ be a finite 0 -sequence of $D$. Then $\operatorname{Rev}(f)$ is a finite 0 -sequence of $D$.

We now state several propositions:

(9) For every finite 0 -sequence $p$ such that $p \neq \emptyset$ there exists a finite 0 sequence $q$ and there exists a set $x$ such that $p=q^{\curlyvee}\langle x\rangle$.

(10) For every natural number $n$ and for every finite 0 -sequence $f$ such that len $f \leq n$ holds $f\lceil n=f$. 
(11) For every finite 0 -sequence $f$ and for all natural numbers $n, m$ such that $n \leq \operatorname{len} f$ and $m \in n$ holds $(f\lceil n)(m)=f(m)$ and $m \in \operatorname{dom} f$.

(12) For every element $i$ of $\mathbb{N}$ and for every finite 0 -sequence $q$ such that $i \leq \operatorname{len} q$ holds len $(q \uparrow i)=i$.

(13) For every element $i$ of $\mathbb{N}$ and for every finite 0 -sequence $q$ holds len $(q\lceil i) \leq$ $i$.

(14) For every finite 0 -sequence $f$ and for every element $n$ of $\mathbb{N}$ such that len $f=n+1$ holds $f=\left(f\lceil n)^{\frown}\langle f(n)\rangle\right.$.

Let $f$ be a finite 0 -sequence and let $n$ be a natural number. The functor $f_{\text {ln }}$ yielding a finite 0 -sequence is defined by:

(Def. 2) $\operatorname{len}\left(f_{\lfloor n}\right)=\operatorname{len} f-{ }^{\prime} n$ and for every natural number $m$ such that $m \in$ $\operatorname{dom}\left(f_{l n}\right)$ holds $f_{\lfloor n}(m)=f(m+n)$.

One can prove the following three propositions:

(15) For every finite 0 -sequence $f$ and for every natural number $n$ such that $n \geq \operatorname{len} f$ holds $f_{\text {ln }}=\emptyset$.

(16) For every finite 0 -sequence $f$ and for every natural number $n$ such that $n<\operatorname{len} f$ holds len $\left(f_{\lfloor n}\right)=\operatorname{len} f-n$.

(17) For every finite 0 -sequence $f$ and for all natural numbers $n, m$ such that $m+n<\operatorname{len} f$ holds $f_{\lfloor n}(m)=f(m+n)$.

Let $f$ be an one-to-one finite 0 -sequence and let $n$ be a natural number. Note that $f_{l n}$ is one-to-one.

We now state several propositions:

(18) For every finite 0-sequence $f$ and for every natural number $n$ holds $\operatorname{rng}\left(f_{l n}\right) \subseteq \operatorname{rng} f$.

(19) For every finite 0 -sequence $f$ holds $f_{l 0}=f$.

(20) For every natural number $i$ and for all finite 0-sequences $f, g$ holds $(f \frown g)_{\lfloor\operatorname{len} f+i}=g_{\lfloor i}$.

(21) For all finite 0-sequences $f, g$ holds $(f \frown g)_{\lfloor\text {len } f}=g$.

(22) For every finite 0 -sequence $f$ and for every element $n$ of $\mathbb{N}$ holds $\left(f\lceil n)^{\frown}\right.$ $\left(f_{l n}\right)=f$.

Let $D$ be a set, let $f$ be a finite 0 -sequence of $D$, and let $n$ be a natural number. Then $f_{l n}$ is a finite 0 -sequence of $D$.

Let $f$ be a finite 0 -sequence and let $k_{1}, k_{2}$ be natural numbers. The functor $\operatorname{mid}\left(f, k_{1}, k_{2}\right)$ yields a finite 0 -sequence and is defined as follows:

(Def. 3) For all elements $k_{11}, k_{21}$ of $\mathbb{N}$ such that $k_{11}=k_{1}$ and $k_{21}=k_{2}$ holds $\operatorname{mid}\left(f, k_{1}, k_{2}\right)=\left(f\left\lceil k_{21}\right)_{\left\lfloor k_{11}-^{\prime} 1\right.}\right.$.

We now state several propositions:

(23) For every finite 0 -sequence $f$ and for all natural numbers $k_{1}, k_{2}$ such that $k_{1}>k_{2}$ holds $\operatorname{mid}\left(f, k_{1}, k_{2}\right)=\emptyset$. 
(24) For every finite 0 -sequence $f$ and for all natural numbers $k_{1}, k_{2}$ such that $1 \leq k_{1}$ and $k_{2} \leq \operatorname{len} f$ holds $\operatorname{mid}\left(f, k_{1}, k_{2}\right)=f_{\mid k_{1}-^{\prime} 1}\left\lceil\left(\left(k_{2}+1\right)-^{\prime} k_{1}\right)\right.$.

(25) For every finite 0 -sequence $f$ and for every natural number $k_{2}$ holds $\operatorname{mid}\left(f, 1, k_{2}\right)=f \nmid k_{2}$.

(26) For every finite 0 -sequence $f$ of $D$ and for every natural number $k_{2}$ such that len $f \leq k_{2}$ holds $\operatorname{mid}\left(f, 1, k_{2}\right)=f$.

(27) For every finite 0 -sequence $f$ and for every element $k_{2}$ of $\mathbb{N}$ holds $\operatorname{mid}\left(f, 0, k_{2}\right)=\operatorname{mid}\left(f, 1, k_{2}\right)$.

(28) For all finite 0 -sequences $f, g$ holds $\operatorname{mid}\left(f^{\frown} g\right.$, len $f+1$, len $\left.f+\operatorname{len} g\right)=g$.

Let $D$ be a set, let $f$ be a finite 0 -sequence of $D$, and let $k_{1}, k_{2}$ be natural numbers. Then $\operatorname{mid}\left(f, k_{1}, k_{2}\right)$ is a finite 0 -sequence of $D$.

Let $f$ be a finite 0 -sequence of $\mathbb{R}$. The functor $\sum f$ yields an element of $\mathbb{R}$ and is defined by the condition (Def. 4).

(Def. 4) There exists a finite 0 -sequence $g$ of $\mathbb{R}$ such that len $f=\operatorname{len} g$ and $f(0)=$ $g(0)$ and for every natural number $i$ such that $i+1<\operatorname{len} f$ holds $g(i+1)=$ $g(i)+f(i+1)$ and $\sum f=g\left(\operatorname{len} f-^{\prime} 1\right)$.

Let $f$ be an empty finite 0 -sequence of $\mathbb{R}$. Observe that $\sum f$ is zero.

We now state two propositions:

(29) For every empty finite 0 -sequence $f$ of $\mathbb{R}$ holds $\sum f=0$.

(30) For all finite 0 -sequences $h_{1}, h_{2}$ of $\mathbb{R}$ holds $\sum h_{1} \frown h_{2}=\left(\sum h_{1}\right)+\sum h_{2}$.

\section{Selected Subsequences}

Let $X$ be a finite natural-membered set. The functor $\operatorname{Sgm}_{0} X$ yields a finite 0 -sequence of $\mathbb{N}$ and is defined as follows:

(Def. 5) $\operatorname{rng} \operatorname{Sgm}_{0} X=X$ and for all natural numbers $l, m, k_{1}, k_{2}$ such that $l<m<\operatorname{len} \operatorname{Sgm}_{0} X$ and $k_{1}=\left(\operatorname{Sgm}_{0} X\right)(l)$ and $k_{2}=\left(\operatorname{Sgm}_{0} X\right)(m)$ holds $k_{1}<k_{2}$.

Let $A$ be a finite natural-membered set. Note that $\operatorname{Sgm}_{0} A$ is one-to-one.

Next we state three propositions:

(31) For every finite natural-membered set $A$ holds $\operatorname{len} \operatorname{Sgm}_{0} A=\overline{\bar{A}}$.

(32) For all finite natural-membered sets $X, Y$ such that $X \subseteq Y$ and $X \neq \emptyset$ holds $\left(\operatorname{Sgm}_{0} Y\right)(0) \leq\left(\operatorname{Sgm}_{0} X\right)(0)$.

(33) For every natural number $n$ holds $\left(\operatorname{Sgm}_{0}\{n\}\right)(0)=n$.

Let $B_{1}, B_{2}$ be sets. The predicate $B_{1}<B_{2}$ is defined by:

(Def. 6) For all natural numbers $n, m$ such that $n \in B_{1}$ and $m \in B_{2}$ holds $n<m$.

Let $B_{1}, B_{2}$ be sets. The predicate $B_{1} \leq B_{2}$ is defined by:

(Def. 7) For all natural numbers $n, m$ such that $n \in B_{1}$ and $m \in B_{2}$ holds $n \leq m$. 
The following propositions are true:

(34) For all sets $B_{1}, B_{2}$ such that $B_{1}<B_{2}$ holds $B_{1} \cap B_{2} \cap \mathbb{N}=\emptyset$.

(35) For all finite natural-membered sets $B_{1}, B_{2}$ such that $B_{1}<B_{2}$ holds $B_{1}$ misses $B_{2}$.

(36) For all sets $A, B_{1}, B_{2}$ such that $B_{1}<B_{2}$ holds $A \cap B_{1}<A \cap B_{2}$.

(37) For all finite natural-membered sets $X, Y$ such that $Y \neq \emptyset$ and there exists a set $x$ such that $x \in X$ and $\{x\} \leq Y$ holds $\left(\operatorname{Sgm}_{0} X\right)(0) \leq$ $\left(\operatorname{Sgm}_{0} Y\right)(0)$.

(38) Let $X_{0}, Y_{0}$ be finite natural-membered sets and $i$ be a natural number. If $X_{0}<Y_{0}$ and $i<\operatorname{card} X_{0}$, then $\operatorname{rng}\left(\operatorname{Sgm}_{0}\left(X_{0} \cup Y_{0}\right)\left\lceil\operatorname{card} X_{0}\right)=X_{0}\right.$ and $\left(\operatorname{Sgm}_{0}\left(X_{0} \cup Y_{0}\right)\left\lceil\operatorname{card} X_{0}\right)(i)=\left(\operatorname{Sgm}_{0}\left(X_{0} \cup Y_{0}\right)\right)(i)\right.$.

(39) For all finite natural-membered sets $X, Y$ and for every natural number $i$ such that $X<Y$ and $i \in \overline{\bar{X}}$ holds $\left(\operatorname{Sgm}_{0}(X \cup Y)\right)(i) \in X$.

(40) Let $X, Y$ be finite natural-membered sets and $i$ be a natural number. If $X<Y$ and $i<\operatorname{len} \operatorname{Sgm}_{0} X$, then $\left(\operatorname{Sgm}_{0} X\right)(i)=\left(\operatorname{Sgm}_{0}(X \cup Y)\right)(i)$.

(41) Let $X_{0}, Y_{0}$ be finite natural-membered sets and $i$ be a natural number. If $X_{0}<Y_{0}$ and $i<\operatorname{card} Y_{0}$, then $\operatorname{rng}\left(\left(\operatorname{Sgm}_{0}\left(X_{0} \cup Y_{0}\right)\right)_{\mid \text {card } X_{0}}\right)=Y_{0}$ and $\left(\operatorname{Sgm}_{0}\left(X_{0} \cup Y_{0}\right)\right)_{\left\lfloor\operatorname{card} X_{0}\right.}(i)=\left(\operatorname{Sgm}_{0}\left(X_{0} \cup Y_{0}\right)\right)\left(i+\operatorname{card} X_{0}\right)$.

(42) Let $X, Y$ be finite natural-membered sets and $i$ be a natural number. If $X<Y$ and $i<\operatorname{len} \operatorname{Sgm}_{0} Y$, then $\left(\operatorname{Sgm}_{0} Y\right)(i)=\left(\operatorname{Sgm}_{0}(X \cup Y)\right)(i+$ len $\left.\operatorname{Sgm}_{0} X\right)$.

(43) For all finite natural-membered sets $X, Y$ such that $Y \neq \emptyset$ and $X<Y$ holds $\left(\operatorname{Sgm}_{0} Y\right)(0)=\left(\operatorname{Sgm}_{0}(X \cup Y)\right)\left(\operatorname{len} \operatorname{Sgm}_{0} X\right)$.

(44) Let $l, m, n, k$ be natural numbers and $X$ be a finite natural-membered set. If $k<l$ and $m<\operatorname{len}^{\operatorname{Sgm}_{0} X}$ and $\left(\operatorname{Sgm}_{0} X\right)(m)=k$ and $\left(\operatorname{Sgm}_{0} X\right)(n)=l$, then $m<n$.

(45) For all finite natural-membered sets $X, Y$ such that $X \neq \emptyset$ and $X<Y$ holds $\left(\operatorname{Sgm}_{0} X\right)(0)=\left(\operatorname{Sgm}_{0}(X \cup Y)\right)(0)$.

(46) For all finite natural-membered sets $X, Y$ holds $X<Y$ iff $\operatorname{Sgm}_{0}(X \cup$ $Y)=\left(\operatorname{Sgm}_{0} X\right) \frown \operatorname{Sgm}_{0} Y$.

Let $f$ be a finite 0 -sequence and let $B$ be a set. The $B$-subsequence of $f$ yields a finite 0 -sequence and is defined as follows:

(Def. 8) The $B$-subsequence of $f=f \cdot \operatorname{Sgm}_{0}(B \cap \operatorname{len} f)$.

One can prove the following proposition

(47) Let $f$ be a finite 0 -sequence and $B$ be a set. Then

(i) len (the $B$-subsequence of $f)=\overline{\overline{B \cap \operatorname{len} f}}$, and

(ii) for every natural number $i$ such that $i<\operatorname{len}$ (the $B$-subsequence of $f$ ) holds (the $B$-subsequence of $f)(i)=f\left(\left(\operatorname{Sgm}_{0}(B \cap \operatorname{len} f)\right)(i)\right)$. 
Let $D$ be a set, let $f$ be a finite 0 -sequence of $D$, and let $B$ be a set. Then the $B$-subsequence of $f$ is a finite 0 -sequence of $D$.

Let $f$ be a finite 0 -sequence. One can verify that the $\emptyset$-subsequence of $f$ is empty.

Let $B$ be a set. Observe that the $B$-subsequence of $\emptyset$ is empty.

We now state the proposition

(48) Let $B_{1}, B_{2}$ be finite natural-membered sets and $f$ be a finite 0 -sequence of $\mathbb{R}$. Suppose $B_{1}<B_{2}$. Then $\sum$ the $B_{1} \cup B_{2}$-subsequence of $f=\left(\sum\right.$ the $B_{1}$-subsequence of $f$ ) $+\sum$ the $B_{2}$-subsequence of $f$.

\section{REFERENCES}

[1] Grzegorz Bancerek. Cardinal numbers. Formalized Mathematics, 1(2):377-382, 1990.

[2] Grzegorz Bancerek. The fundamental properties of natural numbers. Formalized Mathematics, 1(1):41-46, 1990.

[3] Grzegorz Bancerek. Increasing and continuous ordinal sequences. Formalized Mathematics, 1(4):711-714, 1990.

[4] Grzegorz Bancerek. The ordinal numbers. Formalized Mathematics, 1(1):91-96, 1990.

[5] Czesław Byliński. Functions and their basic properties. Formalized Mathematics, 1(1):55$65,1990$.

[6] Agata Darmochwał. Finite sets. Formalized Mathematics, 1(1):165-167, 1990.

[7] Paul R. Halmos. Lectures on Ergodic Theory. The Mathematical Society of Japan, 1956. No.3.

[8] Krzysztof Hryniewiecki. Basic properties of real numbers. Formalized Mathematics, 1(1):35-40, 1990.

[9] Jarosław Kotowicz. Real sequences and basic operations on them. Formalized Mathematics, 1(2):269-272, 1990.

[10] Karol Pạk. Cardinal numbers and finite sets. Formalized Mathematics, 13(3):399-406, 2005.

[11] Andrzej Trybulec. On the sets inhabited by numbers. Formalized Mathematics, 11(4):341$347,2003$.

[12] Zinaida Trybulec. Properties of subsets. Formalized Mathematics, 1(1):67-71, 1990.

[13] Tetsuya Tsunetou, Grzegorz Bancerek, and Yatsuka Nakamura. Zero-based finite sequences. Formalized Mathematics, 9(4):825-829, 2001.

[14] Edmund Woronowicz. Relations and their basic properties. Formalized Mathematics, 1(1):73-83, 1990. 